\title{
Non-electrical reflective seven- segment numeric display using image splitter (Withdrawal Notice)
}

Keisuke Maehara, Kunio Sakamoto

Keisuke Maehara, Kunio Sakamoto, "Non-electrical reflective seven-segment numeric display using image splitter (Withdrawal Notice)," Proc. SPIE 10817, Optoelectronic Imaging and Multimedia Technology V, 108170P (2 November 2018); doi: $10.1117 / 12.2500027$

SPIE. Event: SPIE/COS Photonics Asia, 2018, Beijing, China 


\section{Non-electrical reflective seven-segment numeric display using image splitter (Withdrawal Notice)}

Keisuke Maehara, and Kunio Sakamoto

Konan Univ. (Japan)

Proc. SPIE 10817, 108170P (2018)

Online Publication Date: 2 November 2018

Withdrawn from Publication: 4 February 2019

Conference Date: 11 October-12 October 2018

Conference Location: Beijing, China

Conference Title: Optoelectronic Imaging and Multimedia Technology V

Conference Chairs: Qionghai Dai, Tsutomu Shimura

Publisher's Note: This manuscript, originally published on 2 November 2018, has been withdrawn by the publisher for editorial reasons. 